TRANSACTIONS OF THE

AMERICAN MATHEMATICAL SOCIETY

Volume 350, Number 10, October 1998, Pages 3923-3941

S 0002-9947(98)01973-4

\title{
LOCAL AND GLOBAL PROPERTIES OF LIMIT SETS OF FOLIATIONS OF QUASIGEODESIC ANOSOV FLOWS
}

\author{
SÉRGIO R. FENLEY
}

\begin{abstract}
A nonsingular flow is quasigeodesic when all flow lines are efficient in measuring distances in relative homotopy classes. We analyze the quasigeodesic property for Anosov flows in 3-manifolds which have negatively curved fundamental group. We show that this property implies that limit sets of stable and unstable leaves (in the universal cover) vary continuously in the sphere at infinity. It also follows that the union of the limit sets of all stable (or unstable) leaves is not the whole sphere at infinity. Finally, under the quasigeodesic hypothesis we completely determine when limit sets of leaves in the universal cover can intersect. This is done by determining exactly when flow lines in the universal cover share an ideal point.
\end{abstract}

\section{INTRODUCTION}

This paper concerns the study of the global structure of Anosov flows in 3manifolds. If the fundamental group of the manifold is solvable then the flow is topologically conjugate to a suspension of an Anosov flow as proved by Plante [P12], [P13] (see also [Ba1]). When the manifold is a Seifert fibered space (SFS), Ghys [Gh] proved that the flow is, up to finite covers, topologically conjugate to the geodesic flow in the unit tangent bundle of a hyperbolic surface. If the manifold is a graph manifold, the flow is also fairly well understood [Ba2]. The remaining case to be understood is when there are some pieces of the torus decomposition of the manifold which are not Seifert fibered, but have infinite fundamental group (one cannot have finite fundamental group in the presence of an Anosov flow). When such a piece is not the whole manifold, it is hyperbolic by Thurston's geometrization theorem [Th2]. When such a piece is the whole manifold, it was also conjectured by Thurston that the manifold is hyperbolic [Th2].

Given this, it is natural to try to understand Anosov flows in closed hyperbolic 3 -manifolds. So far this is a very hard problem. There are many examples of Anosov flows in hyperbolic 3-manifolds obtained by Dehn surgery on closed orbits of suspensions or geodesic flows [Go], [Ch], but very little is known about them. In particular, almost nothing is known about their global structure. The main difficulty is that in the SFS case, the fundamental group has a distinguished cyclic normal subgroup, which forces a rigid structure to the flow; and a similar situation occurs for manifolds with solvable fundamental group and for graph manifolds. There is no such structure in the fundamental group of hyperbolic 3-manifolds. In

Received by the editors December 18, 1995 and, in revised form, November 11, 1996.

1991 Mathematics Subject Classification. Primary 57R30, 58F25, 58F15; Secondary 58F22, $53 \mathrm{C} 12$.

Reseach supported by NSF grants DMS-9201744 and an NSF postdoctoral fellowship. 
addition, when considering the geometry, hyperbolic manifolds are locally isotropic, as opposed to SFS.

In order to analyse Anosov flows in hyperbolic 3-manifolds we try to relate them to various fruitful tools in hyperbolic geometry. The standard strategy for studying objects in hyperbolic 3-manifolds is to lift to the universal cover, and then there is a rich structure available. The universal cover is hyperbolic 3 -space $\left(\mathbf{H}^{3}\right)$ which is compactified with a sphere at infinity $\left(S_{\infty}^{2}\right)$, producing a closed 3 -ball. It is of fundamental importance for hyperbolic 3-manifolds to understand the interplay between objects in $\mathbf{H}^{3}$ and their accumulation points in $S_{\infty}^{2}$ (their limit sets). Following this philosophy, we consider orbits of the flow lifted to the universal cover and in particular their asymptotic behavior, that is, how they approach the sphere at infinity. By the shadow lemma [Bo1], [Bo2], such orbits are always properly embedded in $\mathbf{H}^{3}$, so there are two fundamental questions to ask concerning such orbits: The first question is whether flow lines extend continuously to the sphere at infinity. The second, stronger question is about the quasigeodesic behavior of flow lines, which will be the main hypothesis in this article.

Recall that a curve is a quasigeodesic if it is efficient up to a bounded multiplicative distortion in measuring distance in relative homotopy classes [Th1]. A flow is quasigeodesic if all flow lines are quasigeodesics. In hyperbolic manifolds quasigeodesics have the fundamental property of being a bounded distance from true geodesics (in the universal cover) [Th1]. This makes them extremely important, as was demonstrated by Thurston [Th1], [Th2], [Mor] in the proof of the hyperbolization theorem for Haken manifolds.

In this article we will assume the quasigeodesic hypothesis for an Anosov flow in a hyperbolic manifold, and then see the consequences for the limit sets of lifts to the universal cover of the stable and unstable leaves associated to the flow. We stress that at this point there are no known examples of quasigeodesic Anosov flows in hyperbolic 3-manifolds, and in fact one fundamental question is whether it is at all possible that such flows are ever quasigeodesic. Our strategy is to assume the quasigeodesic hypothesis to get an idea of how a quasigeodesic flow would look. In fact this procedure can be used to guess properties of general Anosov flows. This was done very successfully in [Fe4]: the quasigeodesic hypothesis produced results about the topological structure of the stable foliation in the universal cover (of particular interest are its non-Hausdorff points), and much later the same results were proved in [Fe8] without assuming quasigeodesic and even without assuming that the manifold is hyperbolic (or flow transitive, for that matter). This had many important consequences: for instance a characterization of suspensions of Anosov flows up to topological conjugacy as the only flows which do not have (nontrivial) free homotopies between closed orbits of the flow [Fe8]. In addition, this detailed study of the quasigeodesic hypothesis may in fact prove that there are no such flows. To sum it up: the question of whether there are quasigeodesic Anosov flows in hyperbolic 3-manifolds is still very much undecided - a very important and hard question.

From another perspective, notice that there are many examples of quasigeodec pseudo-Anosov flows in hyperbolic manifolds. Pseudo-Anosov flows are (roughly) those flows which have Anosov behavior, except that one allows $p$-prong singularities along finitely many closed orbits. The known examples are: (1) Suspensions of pseudo-Anosov homeomorphisms of surfaces [Ca-Th]. (2) If the second homology of the manifold is nontrivial then there are many quasigeodesic pseudo-Anosov flows 
almost transverse to finite depth foliations [Fe-Mo]. The quasigeodesic property is very useful and was used by Mosher to study Thurston norms of the homology in [Mo1], [Mo2], [Mo3]. The proof of result (2) is constructive: such manifolds have incompressible surfaces, sutured manifold hierarchies and Reebless foliations, and the flows are (almost) tranverse to such foliations. This is one big difficulty in understanding Anosov flows, because they can never be transverse to closed hyperbolic surfaces. In addition there are many examples of Anosov flows in nonHaken hyperbolic manifolds. The whole difference between Anosov and pseudoAnosov flows is the freedom allowed by the introduction of prongs, which makes pseudo-Anosov flows very flexible. This difference is fundamental for the study of the quasigeodesic property (and for many others too). Still there are a lot of similarities between Anosov and pseudo-Anosov flows. For instance, many of the results of this article most likely will hold for quasigeodesic pseudo-Anosov flowseven though this will depend on understanding the local structure of branching for the stable/unstable foliations of a pseudo-Anosov flow in the universal cover - not a trivial matter.

So let $\Phi$ be an Anosov flow in a 3-manifold $M$ with negatively curved fundamental group $[\mathrm{Gr}]$. These are generalizations of hyperbolic 3-manifolds, where many of the same metric constructions work just as well [Gh-Ha]. The universal cover $\widetilde{M}$ is homeomorphic to $\mathbf{R}^{3}$ and is compactified with a sphere at infinity $\left(S_{\infty}^{2}\right)$. The stable and unstable foliations of the Anosov flow $\Phi$ in $M$ lift to two dimensional foliations by topological planes in $\widetilde{M}$, which are denoted by $\widetilde{\mathcal{F}}^{s}$ and $\widetilde{\mathcal{F}}^{u}$. We proved in [Fe3] that the quasigeodesic property for $\Phi$ implies that leaves of $\widetilde{\mathcal{F}}^{s}$ and $\widetilde{\mathcal{F}}^{u}$ extend continuously to $S_{\infty}^{2}$, giving continuous parametrizations of their limit sets. We remark that this continuous extension property can be defined for any Reebless foliation in a 3-manifold with negatively curved fundamental group, and it has been proved for fibrations [Ca-Th] and for many depth one foliations [Fe1].

We say that $\Phi$ is $\mathbf{R}$-covered if one of $\widetilde{\mathcal{F}}^{s}$ or $\widetilde{\mathcal{F}}^{u}$ has Hausdorff leaf space, in which case both leaf spaces are homeomorphic to the set $\mathbf{R}$ of real numbers [Fe3]. Notice that we proved in [Fe3] (see also [Fe4]) that if $\Phi$ is $\mathbf{R}$-covered then $\Phi$ is not quasigeodesic. When $\Phi$ is $\mathbf{R}$-covered it also follows that the limit set of every leaf $F$ in $\widetilde{\mathcal{F}}^{s}$ or $\widetilde{\mathcal{F}}^{u}$ is the whole sphere. Almost nothing is known about limit sets in the non-Hausdorff case. The purpose of this article is to analyse these limit sets. We show that the quasigeodesic hypothesis has strong consequences for the structure of the limit sets. We remark here that (in the case of a hyperbolic ambient manifold) there are many examples of Anosov flows where the leaf space of $\widetilde{\mathcal{F}}^{s}$ is Hausdorff [Fe3] and also many examples where it is not Hausdorff [Fe8]. In [Fe8] we analysed the limit set of a single leaf and showed that if $\Phi$ is not $\mathbf{R}$-covered and leaves of $\widetilde{\mathcal{F}}^{s}$ and $\widetilde{\mathcal{F}}^{u}$ have the continuous extension property, then for any leaf $F$, its limit set is a Sierpinski set.

In this article "global" is used to refer to the collection of all limit sets of leaves, and "local" refers to how limit sets of nearby leaves are related and the intersection of distinct limit sets. Our first result shows that the quasigeodesic hypothesis implies that the union of all limit sets is not the whole sphere:

Theorem A. Let $\Phi$ be an Anosov flow in $M^{3}$ with negatively curved fundamental group. Suppose that $\Phi$ is quasigeodesic. Then there is a group invariant subset $\mathcal{E}$ of $S_{\infty}^{2}$ consisting of fixed points of covering translations of $M$ and so that, for every leaf $F$ of $\widetilde{\mathcal{F}}^{s}$ or $\widetilde{\mathcal{F}}^{u}$, the limit set of $F$ is disjoint from $\mathcal{E}$. 
We then analyse how limit sets change as the leaves vary. We show that quasigeodesic behavior implies a continuous relation:

Theorem B. Let $\Phi$ be an Anosov flow in $M^{3}$ with negatively curved fundamental group. Suppose that $\Phi$ is quasigeodesic. Then the limit set $\Lambda_{F}$, as a function of $F \in \widetilde{\mathcal{F}}^{s}$, forms a continuous map into the set of all closed sets of $S_{\infty}^{2}$ with the Hausdorff topology.

Finally we analyse the situations in which limit sets of different leaves intersect. This provides a picture of the geography of the collection of the limit sets in $S_{\infty}^{2}$. Since leaves extend continuously to $S_{\infty}^{2}$, all limit points of $F \in \widetilde{\mathcal{F}}^{s}$ are obtained from ideal points of flow lines in $F$. Therefore this boils down to understanding when flow lines have an ideal point in common. Given $F \in \widetilde{\mathcal{F}}^{s}$, let $\eta_{+}(F)$ be the common forward limit point of all flow lines in $F$ (which are forward asymptotic), and similarly define $\eta_{-}(G)$ for $G \in \widetilde{\mathcal{F}}^{u}$. Then we are lead to understand when the equality $\eta_{+}(F)=\eta_{+}(L)$ holds, for $F, L \in \widetilde{\mathcal{F}}^{s}$. The case $\eta_{+}(F)=\eta_{-}(G)$ can be reduced to this one; see section 5 . We prove:

Theorem C. Let $\Phi$ be a quasigeodesic Anosov flow in $M^{3}$ with negatively curved fundamental group. Let $F, L \in \widetilde{\mathcal{F}}^{s}$ be such that $\eta_{+}(F)=\eta_{+}(L)$. Then one of the following alternatives happens:

(1) $F=L$;

(2) $F \neq L$. Then $F, L$ are the extremities of a finite chain of stable or unstable leaves, so that consecutive leaves in the chain are either in the same foliation and are not separated from each other (in the leaf space of their foliation), or are in distinct foliations and are "asymptotic" to each other. In general if any leaf of the chain is periodic, then all leaves in the collection are periodic and left invariant by a common nontrivial covering translation. In particular this happens if there is a pair of consecutive leaves in the chain which are not separated from each other.

Finally the converse is also true, that is, if $F, L \in \widetilde{\mathcal{F}}^{s}$ satisfy (1) or (2), then $\eta_{+}(F)=\eta_{+}(L)$.

We refer the reader to section 5 for a detailed explanation of the terms and conditions in this result.

The key fact repeatedly used in proving all of these results is that if the flow is quasigeodesic, then knowledge of a limit point of a flow line implies a good knowledge of where this flow line is. This cannot be assured when one only assumes the continuous extension of leaves of $\widetilde{\mathcal{F}}^{s}, \widetilde{\mathcal{F}}^{u}$ to $S_{\infty}^{2}$.

As the proofs of the theorems will show, all these properties of limit sets are strongly related to the topological structure of the foliations $\widetilde{\mathcal{F}}^{s}, \widetilde{\mathcal{F}}^{u}$ in $\widetilde{M}$. Therefore the geometry (that is, the limit sets) is reflected by the topological structure of the flow.

We also prove the following result, which is of independent interest. There are no assumptions on the manifold or on the metric properties of flow lines.

Theorem D. Let $\Phi$ be a transitive Anosov flow in $M^{3}$. Then there are many elements of the fundamental group of $M$ which cannot be represented by closed orbits of $\Phi$. 


\section{Preliminaries}

Let $\Phi_{t}: M^{3} \rightarrow M^{3}$ be a nonsingular flow in a closed Riemannian manifold $M^{3}$. The flow $\Phi$ is Anosov if there is a continuous decomposition of the tangent bundle $T M$ as a Whitney sum $T M=E^{0} \oplus E^{s} \oplus E^{u}$ of $D \Phi_{t}$ invariant one dimensional subbundles and there are constants $\mu_{0} \geq 1, \mu_{1}>0$ so that:

i) $E^{0}$ is tangent to the flow,

ii) $\left\|D \Phi_{t}(v)\right\| \leq \mu_{0} e^{-\mu_{1} t}\|v\|$ for any $v \in E^{s}, t \geq 0$,

iii) $\left\|D \Phi_{-t}(v)\right\| \leq \mu_{0} e^{-\mu_{1} t}\|v\|$ for any $v \in E^{u}, t \geq 0$.

The foliations $\mathcal{F}^{s s}, \mathcal{F}^{u u}$, called strong stable $\left(\mathcal{F}^{s s}\right)$ and strong unstable $\left(\mathcal{F}^{u u}\right)$, are obtained by integrating $E^{s}$ and $E^{u}$. The bundles $E^{0} \oplus E^{s}$ and $E^{0} \oplus E^{u}$ are also integrable [An], producing 2-dimensional foliations $\mathcal{F}^{s}, \mathcal{F}^{u}$ which are the stable and unstable foliations of the flow.

The leaves of $\mathcal{F}^{s}, \mathcal{F}^{u}$ are topological planes, annuli, or Möebius bands. The last two correspond exactly to leaves containing closed orbits of $\Phi$. The flow is transitive if the periodic orbits form a dense subset of $M$. Equivalently, every leaf of $\mathcal{F}^{s}$ or $\mathcal{F}^{u}$ is dense $[\mathrm{An}],[\mathrm{Pl} 1]$.

Let $\pi: \widetilde{M} \rightarrow M$ be the universal covering space of $M$. This notation will be fixed throughout the article. The Anosov foliations $\mathcal{F}^{s}, \mathcal{F}^{u}$ lift to foliations by topological planes $\widetilde{\mathcal{F}}^{s}, \widetilde{\mathcal{F}}^{u}$ in $\widetilde{M}$ [No]. Hence $\widetilde{M}$ is homeomorphic to $\mathbf{R}^{3}[\mathrm{~Pa}]$ and $M$ is irreducible. The induced flow in $\widetilde{M}$ is denoted by $\widetilde{\Phi}$.

Let $\mathcal{O}$ be the orbit space of $\widetilde{\Phi}$ obtained by collapsing flow lines to points. An important property which will be often used is that $\mathcal{O}$ is Hausdorff and therefore homeomorphic to $\mathbf{R}^{2}$ [Fe3]. $\widetilde{\mathcal{F}}^{s}, \widetilde{\mathcal{F}}^{u}$ induce two transverse 1-dimensional foliations in $\mathcal{O}$. Occasionally we will identify sets in $\widetilde{M}$ or orbits of $\widetilde{\Phi}$ with their respective images in $\mathcal{O}$.

Let $W^{s}(x)$ be the leaf of $\mathcal{F}^{s}$ containing $x$. In the same way we define the leaves $W^{u}(x), W^{s s}(x), W^{u u}(x), \widetilde{W}^{s}(x), \widetilde{W}^{u}(x), \widetilde{W}^{s s}(x)$ and $\widetilde{W}^{u u}(x)$.

If $L$ is a leaf of $\widetilde{\mathcal{F}}^{s}$ or $\widetilde{\mathcal{F}}^{u}$, then a half leaf of $L$ is a connected component of $L-\gamma$, where $\gamma$ is any full orbit in $L$. If $\Phi$ is transitive, then the image of any half leaf of $\mathcal{F}^{s}$ or $\mathcal{F}^{u}$ in $M$ is dense in $M$ [Fe3].

Since $\widetilde{M}$ is simply connected, it follows that $\widetilde{\mathcal{F}}^{s}, \widetilde{\mathcal{F}}^{u}$ are always transversely orientable. We fix transversal orientations. For $p \in \widetilde{M}$, let $\widetilde{W}_{+}^{s}(p)$ be the half leaf of $\widetilde{W}^{s}(p)$ defined by $\widetilde{\Phi}_{\mathbf{R}}(p)$ and the positive transversal orientation to $\widetilde{\mathcal{F}}^{u}$ at $p$. Similarly define $\widetilde{W}_{-}^{s}(p), \widetilde{W}_{+}^{u}(p)$ and $\widetilde{W}_{-}^{u}(p)$. If $L \in \widetilde{\mathcal{F}}^{s} \cup \widetilde{\mathcal{F}}^{u}$, then the front (back) side of $L$ is the component of $\widetilde{M}-L$ defined by the positive (negative) transversal orientation to $L$.

General references for Anosov flows are [An] and [Sm].

\section{LIMIT POINTS OF FLOW LINES}

We first prove that in general there are many distinguished covering translations of $\widetilde{M}$ which are not associated to closed orbits of the flow $\Phi$. There are no assumptions on the manifold and no quasigeodesic assumptions on the flow.

A branching pair $F, L \in \widetilde{\mathcal{F}}^{s}$ (or $\widetilde{\mathcal{F}}^{u}$ ) is a pair of leaves not separated in the respective leaf space. We say that a branching pair $F, L$ produces branching in the positive direction if they are not separated on their negative sides. Similarly we define branching in the negative direction. 


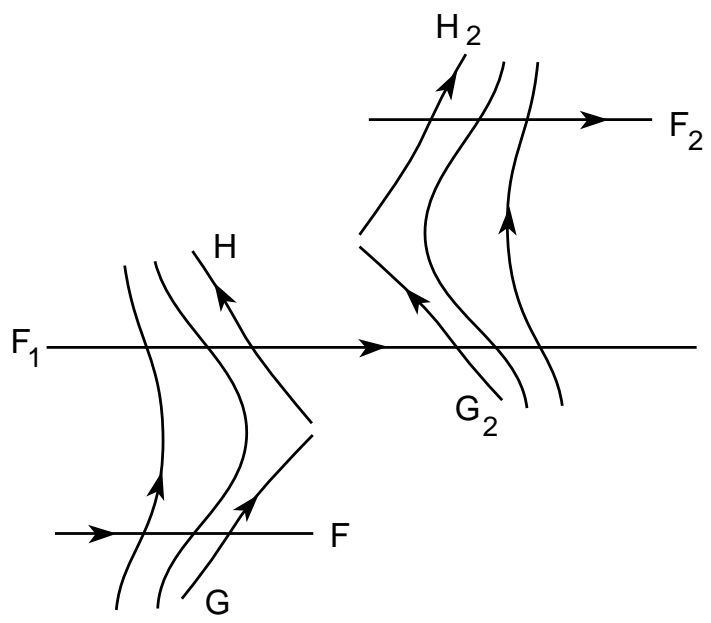

FiguRE 1. Covering translations separating leaves.

A leaf $L$ of $\widetilde{\mathcal{F}}^{s}$ or $\widetilde{\mathcal{F}}^{u}$ is invariant under a covering translation $g$ if $g(F)=F$. It is almost invariant under $g$ if $F$ and $g(F)$ are not separated in the respective leaf space.

Proposition 3.1. Let $\Phi$ be a transitive, non-R-covered Anosov flow in $M^{3}$. Let $F \in \widetilde{\mathcal{F}}^{s}$ and let $g$ be a covering translation preserving the transversal orientations to $\widetilde{\mathcal{F}}^{s}, \widetilde{\mathcal{F}}^{u}$ and satisfying: $F$ and $g(F)$ do not intersect a common unstable leaf and either $g(F)$ is in front of $F$ and $F$ is in back of $g(F)$ or vice-versa. Then $g$ has neither invariant nor almost invariant leaves. Therefore $g$ is not associated to a closed orbit of $\Phi$. In addition, for any $F \in \widetilde{\mathcal{F}}^{s}$ there is a $g$ satisfying the above hypothesis.

Proof. By taking a finite cover if necessary we may assume that $\mathcal{F}^{s}, \mathcal{F}^{u}$ are transversely orientable. Therefore any covering translation preserves the transversal orientations to $\widetilde{\mathcal{F}}^{s}, \widetilde{\mathcal{F}}^{u}$. This fact will often be implicitly used in this and other subsequent proofs.

Since $\Phi$ is not $\mathbf{R}$-covered and $\Phi$ is transitive, the main theorem of [Fe7] implies that both $\widetilde{\mathcal{F}}^{s}$ and $\widetilde{\mathcal{F}}^{u}$ have branching in the positive and negative directions. Let $F \in \widetilde{\mathcal{F}}^{s}$ and let $G^{\prime}, H^{\prime} \in \widetilde{\mathcal{F}}^{u}$, which are not separated in their negative sides. After switching $G^{\prime}$ and $H^{\prime}$ if necessary we may assume that there are $E_{1}, E_{2} \in \widetilde{\mathcal{F}}^{s}$ with $E_{2}$ in front of $E_{1}$ and $G^{\prime} \cap E_{1} \neq \emptyset, H^{\prime} \cap E_{2} \neq \emptyset$. Since $\Phi$ is transitive, there is a translate $G$ of $G^{\prime}$ with $G \cap F \neq \emptyset$. The corresponding translate $H$ of $H^{\prime}$ is not separated from $G$ and is in front of $F$ (see fig. 1). Let $F_{1} \in \widetilde{\mathcal{F}}^{s}$ with $H \cap F_{1} \neq \emptyset$.

Now let $G_{2}, H_{2} \in \widetilde{\mathcal{F}}^{u}$ be not separated in their positive sides and so that $G_{2}$ is in front of $H, G_{2} \cap F_{1} \neq \emptyset$, and there is $F_{2} \in \widetilde{\mathcal{F}}^{s}, F_{2}$ in front of $F_{1}$ and $H_{2} \cap F_{2} \neq \emptyset$; see fig. 1. Since $G$ and $H$ are not separated, there is a transversal to $\widetilde{\mathcal{F}}^{s}$ from $F$ to $F_{1}$ and by the same reason a transversal from $F_{1}$ to $F_{2}$.

Choose a translate $g(F) \cap H_{2} \neq \emptyset$. Hence there is a transversal from $F$ to $g(F)$. Notice that $H$ separates $F$ from $g(F)$; therefore $F$ and $g(F)$ do not intersect a common unstable leaf. 
From now on, all we need is that $g$ is a covering translation for which there is some $F \in \widetilde{\mathcal{F}}^{s}$ with $g(F)$ in front of $F$ and $F$ in back of $g(F)$ and so that $F$ and $g(F)$ do not intersect a common unstable leaf.

For any $n \in \mathbf{Z}$ it follows that $g^{n}(F)$ is in the back of $g^{n+1}(F)$ and $g^{n+1}(F)$ is in the front of $g^{n}(F)$. Let $\mathcal{C}_{n}$ be the back of $g^{n}(F)$. Suppose that $\mathcal{C}=\bigcup_{n \in \mathbf{N}} \mathcal{C}_{n} \neq \widetilde{M}$. Then $\mathcal{C}$ is an open, connected, $\widetilde{\mathcal{F}}^{s}$ saturated proper subset of $\widetilde{M}$. Let $L$ be a leaf in $\partial \mathcal{C}$, and let $V \in \widetilde{\mathcal{F}}^{u}$ with $V \cap L \neq \emptyset$. Then, for $n$ big enough, $g^{n}(F) \cap V \neq \emptyset$. Hence $g^{n}(F)$ and $g^{n+1}(F)$ intersect $V$, implying $F$ and $g(F)$ intersect $g^{-n}(V)$-a contradiction.

This implies that for any $L \in \widetilde{\mathcal{F}}^{s}$ there is a unique $n \in \mathbf{Z}$ so that either $L=g^{n}(F)$ or $L$ is in front of $g^{n}(F)$ and in back of $g^{n+1}(F)$. In the first case $L$ is clearly separated from $g(L)$. In the second case $g^{n+1}(F)$ separates $L$ from $g(L)$. We conclude that there are no almost invariant leaves of $\widetilde{\mathcal{F}}^{s}$ under $g$.

Finally, if $g$ is associated to the closed orbit $\gamma$ of $\Phi$, then there is a lift $F$ of $W^{s}(\gamma)$ so that $g(F)=F$. This contradicts the above arguments and finishes the proof.

Remarks. (1) The fact that $g$ has no almost invariant leaves (which implies no invariant leaves also) shows that the action of $g$ in the leaf space of $\widetilde{\mathcal{F}}^{s}$ has an invariant axis [Ba2], which can be shown to be homeomorphic to the real line. Using results of [Fe8], one can then show that the action on the leaf space of $\widetilde{\mathcal{F}}^{u}$ also has no almost invariant leaves. The invariant axis for the action in $\widetilde{\mathcal{F}}^{u}$ will not be Hausdorff.

(2) It is easy to prove proposition 3.1 for $\mathbf{R}$-covered Anosov flows, which are not topologically conjugate to suspensions of Anosov flows. More precisely, given the topological description of $\mathbf{R}$-covered Anosov flows in [Fe3], it follows that proposition 3.1 holds for any transitive Anosov flow, so that there is a leaf of $\widetilde{\mathcal{F}}^{s}$ which does not intersects every leaf of $\widetilde{\mathcal{F}}^{u}$. Barbot showed that, up to topological conjugacy, suspensions are the only flows which fail this last condition [Ba1].

We assume from now on that $\pi_{1}\left(M^{3}\right)$ is negatively curved [Gr]. Gromov showed that there is a canonical compactification of $\widetilde{M}$ with an ideal boundary $\partial \widetilde{M}$. Since $M$ is irreducible, Bestvina and Mess [Be-Me] proved that $\partial \widetilde{M}$ is homeomorphic to a sphere, denoted by $S_{\infty}^{2}$. Furthermore, $\widetilde{M} \cup S_{\infty}^{2}$ is homeomorphic to the closed 3-ball [Be-Me]. Basic references for negatively curved groups are [Gr], [Gh-Ha].

Sullivan $[\mathrm{Su}]$ proved that the intrinsic geometry of the leaves of $\widetilde{\mathcal{F}}^{s}$ and $\widetilde{\mathcal{F}}^{u}$ is negatively curved in the large [Gr]. Then any leaf $L \in \widetilde{\mathcal{F}}^{s} \cup \widetilde{\mathcal{F}}^{u}$ also has a canonical compactification with an intrinsic ideal boundary $\partial_{\infty} L[\mathrm{Gr}]$. We proved in [Fe1] that $\partial_{\infty} L$ is always homeomorphic to a circle, denoted by $S_{\infty}^{1}$, usually without reference to the particular leaf we are considering. We stress that $S_{\infty}^{1}$ will always be the intrinsic ideal boundary of $L$ and not its image in $\partial \widetilde{M}$ when the leaf extends continuously to $\partial \widetilde{M}$. Furthermore, $S_{\infty}^{1}$ is defined independently of whether $\Phi$ is quasigeodesic or not.

If $L \in \widetilde{\mathcal{F}}^{s}$, then the intrinsic ideal points correspond to the (distinct) negative limit points of flow lines in $L$ and to the common positive point of all flow lines [Fe3]. The intrinsic geometry of $L \in \widetilde{\mathcal{F}}^{s}$ resembles that of the hyperbolic plane $\mathbf{H}^{2}$, where the flow lines correspond to the geodesics in $\mathbf{H}^{2}$ which have a common limit point in the ideal boundary of $\mathbf{H}^{2}$. Analogous results hold for $\widetilde{\mathcal{F}}^{u}$. 
Definition 3.2. A quasi-isometry is a map $\rho:(Q, d) \rightarrow\left(Q^{\prime}, d^{\prime}\right)$ between metric spaces for which there is $k \geq 1$ so that, for any $x, y \in Q$,

$$
\begin{aligned}
& \max (d(\rho(x), \rho(y)), d(x, y))>k \\
& \quad \Rightarrow \frac{1}{k} d(x, y) \leq d(\rho(x), \rho(y)) \leq k d(x, y) .
\end{aligned}
$$

Then we say that $\rho$ is a $k$-quasi-isometry.

Definition 3.3. A quasigeodesic curve in $Q$ is the projection to $Q$ of the image of a quasi-isometric embedding $\rho: A \rightarrow \widetilde{Q}$, where $A$ is an interval in $\mathbf{R}$, finite or not, and $\widetilde{Q}$ is the universal cover of $Q$. The metric in $A$ is induced from arc length in $\widetilde{Q}$. A nonsingular flow in $Q$ is quasigeodesic if all flow lines are quasigeodesics. A $k$-quasigeodesic is the image (in $Q$ ) of a $k$-quasi-isometry.

Given a bi-infinite $k$-quasigeodesic in $M$, any lift to $\widetilde{M}$ is a bounded distance from a minimal geodesic of $\widetilde{M}$ [Gr]. The bound depends only on $k$ and how much $\pi_{1}(M)$ is negatively curved. As a result the quasigeodesic has two distinct limit points in $S_{\infty}^{2}$ [Gr, section 7.2]. Infinite quasigeodesic rays also have a well defined limit point.

Definition 3.4. The limit set of a subset $B$ of $\widetilde{M}$ is $\Lambda_{B}=\bar{B} \cap S_{\infty}^{2}$, where the closure is taken in $\widetilde{M} \cup S_{\infty}^{2}$.

Usually $B$ will be a leaf of $\widetilde{\mathcal{F}}^{s}$ or $\widetilde{\mathcal{F}}^{u}$.

In [Fe3] we proved that $\Phi$ being quasigeodesic in $M^{3}$ with $\pi_{1}(M)$ negatively curved implies the following: if $L$ is a leaf of $\widetilde{\mathcal{F}}^{s} \cup \widetilde{\mathcal{F}}^{u}$ then the embedding $\phi: L \rightarrow \widetilde{M}$ extends continuously to $\phi: L \cup S_{\infty}^{1} \rightarrow \widetilde{M} \cup S_{\infty}^{2}$. In particular, $\Lambda_{L}=\phi\left(S_{\infty}^{1}\right)$, giving a continuous parametrization of the limit set of each leaf. If this weaker property happens for all leaves of $\widetilde{\mathcal{F}}^{s}$ and $\widetilde{\mathcal{F}}^{u}$, we say that $\Phi$ (or $\widetilde{\mathcal{F}}^{s}, \widetilde{\mathcal{F}}^{u}$ ) has the continuous extension property.

In [Fe3] we show that if $\Phi$ has the continuous extension property (in particular, when $\Phi$ is quasigeodesic), then there is a continuous function

$$
\eta_{+}: \widetilde{M} \rightarrow S_{\infty}^{2}, \quad \eta_{+}(x)=\lim _{t \rightarrow \infty} \widetilde{\Phi}_{t}(x) .
$$

The same notation will be used for the corresponding function $\mathcal{O} \rightarrow S_{\infty}^{2}$; that is, $\eta_{+}\left(\widetilde{\Phi}_{\mathbf{R}}(x)\right)=\eta_{+}(x)$. In addition, for any $x, y \in F \in \widetilde{\mathcal{F}}^{s}$, we have $\eta_{+}(x)=\eta_{+}(y)$; hence we denote this by $\eta_{+}(F)$. Similarly define $\eta_{-}$and $\eta_{-}(G)$ for $G \in \widetilde{\mathcal{F}}^{u}$.

Notice that if $\Phi$ is quasigeodesic then $\eta_{+}(x) \neq \eta_{-}(x)$ for any $x \in \widetilde{M}$. This is a fundamental property which does not work in general when only the continuous extension property is assumed, for instance in the case of $\mathbf{R}$-covered Anosov flows [Fe5].

Every Anosov flow in $M^{3}$ with $\pi_{1}(M)$ negatively curved is transitive [Fe4]. Hence if the Anosov flow is quasigeodesic, there is $k>0$ so that all flow lines of $\widetilde{\Phi}$ are uniformly $k$-quasigeodesics. Then all flow lines (in $\widetilde{M}$ ) are a globally bounded distance $R>0$ from corresponding minimal geodesics. We will use the following result:

Theorem 3.5 ([Fe4], [Fe8]). Let $\Phi$ be a quasigeodesic Anosov flow in $M^{3}$ with negatively curved fundamental group. Then

i) $\Phi$ is not $\mathbf{R}$-covered, that is, $\widetilde{\mathcal{F}}^{s}$ and $\widetilde{\mathcal{F}}^{u}$ have branching; 
ii) the limit set of any $F \in \widetilde{\mathcal{F}}^{s} \cup \widetilde{\mathcal{F}}^{u}$ is a Sierpinski curve, that is, the complement of a countable dense union of open disks in $S_{\infty}^{2}$.

Given a covering translation $g$ of $\widetilde{M}$, let $\operatorname{Att}(g) \in S_{\infty}^{2}$ be the attracting fixed point of $g$. Let $\operatorname{Rep}(g)$ be the repelling fixed point of $g$ and let $F i x(g)=\operatorname{Att}(g) \cup \operatorname{Rep}(g)$.

Theorem 3.6. Let $\Phi$ be a quasigeodesic Anosov flow in $M^{3}$ with negatively curved fundamental group. Let $g \in \pi_{1}(M)$. If Fix $(g) \cap \Lambda_{F} \neq \emptyset$ for some $F \in \widetilde{\mathcal{F}}^{s} \cup \widetilde{\mathcal{F}}^{u}$, then the covering translation $g$ corresponds to a closed orbit of $\Phi$. Hence there is a periodic orbit $\zeta$ of $\widetilde{\Phi}$ with $g(\zeta)=\zeta$ and $\eta_{+}(\zeta)=\operatorname{Att}(g), \eta_{-}(\zeta)=\operatorname{Rep}(g)$.

Proof. Let $F \in \widetilde{\mathcal{F}}^{s}$ be such that $\operatorname{Att}(g) \in \Lambda_{F}$, and let $q=\operatorname{Rep}(g)$. Since $p \in \Lambda_{F}$, there is a flow line $\gamma$ of $\widetilde{\Phi}$ in $\subset F$ with either $\eta_{+}(\gamma)=p$ or $\eta_{-}(\gamma)=p$. Suppose $\eta_{+}(\gamma)=p$, the other case being similar. Let $z=\eta_{-}(\gamma) \neq p$. Let $\gamma_{n}=g^{n}(\gamma), n<0$. Then

$$
z_{n}=g^{n}(z)=g^{n}\left(\eta_{-}(\gamma)\right)=\eta_{-}\left(g^{n}(\gamma)\right) \rightarrow q, \text { as } n \rightarrow-\infty
$$

Let $\alpha_{n}$ be a minimal geodesic in $\widetilde{M}$ with endpoints $p, z_{n}$. Since $z_{n} \rightarrow q$ and $\widetilde{M}$ is negatively curved in the large, we may choose a subsequence $\alpha_{n} \rightarrow \alpha$, where $\alpha$ is a minimal geodesic with endpoints $p, q[\mathrm{Gr}]$, [Gh-Ha]. Let $y \in \alpha$. As $d\left(\gamma_{n}, \alpha_{n}\right)<R$, then for any $n$ big we may choose $x_{n} \in \gamma_{n}$ with $d\left(x_{n}, y\right)<R+1$. Up to a further subsequence we may assume that $x_{n} \rightarrow x \in \widetilde{M}$. Then $x \in L \in \widetilde{\mathcal{F}}^{s}$ and

$$
\eta_{+}(x)=p, \eta_{-}(x)=q,
$$

because $\eta_{-}$and $\eta_{+}$are continuous. Let $\beta=\widetilde{\Phi}_{\mathbf{R}}(x)$ and let $\beta_{n}=g^{n}(\beta)$, so $\eta_{-}\left(\beta_{n}\right)=$ $q, \eta_{+}\left(\beta_{n}\right)=p$. Proposition 4.9 of [Fe4] implies that there are only finitely many distinct $\beta_{n}$. Hence there is $m$ with $g^{m}(\beta)=\beta$, which implies that $L=\widetilde{W^{s}}(\beta)$ is periodic under $g, g^{m}(L)=L$. Since $\mathcal{F}^{s}, \mathcal{F}^{u}$ are transversely orientable, proposition 3.1 of [Fe6] implies that $g(L)=L$. Therefore $g$ is associated to a closed loop in $\pi(L)$. But $\pi_{1}(\pi(L))$ is generated by the closed orbit of $\Phi$ in $\pi(L)$; hence $g$ corresponds to a multiple of this closed orbit. This finishes the proof.

Given $u \in S_{\infty}^{2}$ and $F \in \widetilde{\mathcal{F}}^{s}$, we say that the ideal point $u$ is above $F$ if $u \notin \Lambda_{F}$ and there is a neighborhood $U$ of $u$ in $\widetilde{M} \cup S_{\infty}^{2}$ so that $U \cap \widetilde{M}$ is in front of $F$. Similarly we define $u$ is below $F$. If $Z$ is a connected component of $\Lambda_{F}^{c}=S_{\infty}^{2}-\Lambda_{F}$, we say that $Z$ is above $F$ if $Z$ only contains points of $S_{\infty}^{2}$ above $F$. Similarly we define $Z$ below $F$. Notice that any component of $\Lambda_{F}^{c}$ is either above or below $F$.

Corollary 3.7. Let $\Phi$ be a quasigeodesic Anosov flow in $M^{3}$ with negatively curved fundamental group. Let $g \in \pi_{1}(M)$ be such that there is $F \in \widetilde{\mathcal{F}}^{s}$ so that $g(F)$ is in front of $F, F$ is in back of $g(F)$ but $F$ and $g(F)$ do not intersect a common unstable leaf. Then Fix $(g) \cap \Lambda_{L}=\emptyset$ for any $L \in \widetilde{\mathcal{F}}^{s}$ or $\widetilde{\mathcal{F}}^{u}$. The set of fixed points of these covering translations produces a nonempty, $\pi_{1}(M)$ invariant subset of $S_{\infty}^{2}$ which is disjoint from the union of all limit sets of leaves of $\widetilde{\mathcal{F}}^{s}$ and $\widetilde{\mathcal{F}}^{u}$.

Proof. By proposition 3.1, $g$ is not associated to a closed orbit of $\Phi$. The previous theorem implies that $\operatorname{Att}(g) \notin \Lambda_{L}$ for any $L \in \widetilde{\mathcal{F}}^{s}$ or $\widetilde{\mathcal{F}}^{u}$. Hence $\operatorname{Att}(g)$ is either above or below $F$. Let $x \in F$. The $g^{n}(F), n>0$, are all above $F$. But $g^{n}(x) \rightarrow$ $\operatorname{Att}(g)$. This shows that $\operatorname{Att}(g)$ is above $F$. Similarly for $\operatorname{Rep}(g)$. 


\section{LIMIT SETS VARY CONTINUOUSLY}

In this section we show that limit sets of stable leaves vary continuously in the sphere at infinity. Analogous results hold for the unstable foliation.

Definition 4.1 (Hausdorff topology). Let $\mathcal{P}$ be the set of closed subsets of a Hausdorff space $Q$. The Hausdorff topology on $\mathcal{P}$ is defined by: if $B_{i} \in \mathcal{P}$ for $i \in \mathbf{N}$ then $B_{i} \rightarrow B \in \mathcal{P}$ if the following 2 conditions are satisfied:

(i) For any $x \in B$ there is a sequence $\left\{x_{i}\right\}_{i \in \mathbf{N}}$ with $x_{i} \in B_{i}$ and $x_{i} \rightarrow x$ as $i \rightarrow+\infty$.

(ii) Let $x_{j} \in B_{i_{j}}$, where $i_{j} \rightarrow \infty$ when $j \rightarrow \infty$. If $x_{j}$ is a convergent sequence in $Q$, then its limit is in $B$.

When $(Q, d)$ is a compact metric space, the geometric topology on $\mathcal{P}$ is the same as the topology given below. For any set $A \subset Q$ and $\epsilon>0$, let

$$
N(A, \epsilon)=\{x \in Q \mid d(A, x)<\epsilon\} .
$$

For any subsets $A, B$ of $M$ define

$$
d(A, B)=\inf _{\epsilon>0}\{\epsilon \mid A \subset N(B, \epsilon) \text { and } B \subset N(A, \epsilon)\} .
$$

Then $d$ is a metric in $\mathcal{P}(M)$ defining the Hausdorff topology.

Our goal is to show that the limit sets $\Lambda_{F}, F \in \widetilde{\mathcal{F}}^{s}$, vary continuously in the Hausdorff topology. Since the leaf space of $\widetilde{\mathcal{F}}^{s}$ is usually not Hausdorff, we will specify what we mean by continuity of limit sets.

We say that $F_{i} \in \widetilde{\mathcal{F}}^{s}$ converges to $L \in \widetilde{\mathcal{F}}^{s}$, if for any neighborhood $U$ of $L$ one has $F_{i} \in U$ for big enough $i$. The total limit of a convergent sequence is the union of all limits of the sequence and is denoted by $\mathcal{T} L\left(F_{i}\right)$. A sequence is monotone if either for all $i$ big enough $F_{i}$ is in front of $F_{i-1}$ or for all $i$ big enough $F_{i}$ is in back of $F_{i-1}$.

The structure of the set $\mathcal{B}_{L}$ of leaves not separated from $L \in \widetilde{\mathcal{F}}^{s}$ and on a given side of $L$ is as follows [Fe8]: Let $E^{\prime}, E^{\prime \prime} \in \mathcal{B}_{L}$. Let $G^{\prime}, G^{\prime \prime} \in \widetilde{\mathcal{F}}^{u}$ with $G^{\prime} \cap E^{\prime} \neq \emptyset$, $G " \cap E " \neq \emptyset$. We say that $E^{\prime}<E^{\prime \prime}$ in $\mathcal{B}_{L}$ if $G^{\prime}$ is in the back of $G$ ". We proved in [Fe8] that with this order $\mathcal{B}_{L}$ is either finite or isomorphic to $\mathbf{Z}$. The notation is $\mathcal{B}_{L}=\left\{L_{j}\right\}_{j \in J \subset \mathbf{Z}}$, where $L_{i}<L_{j}$ if $i<j$. This works irrespective of $M$ being hyperbolic or not.

When $\pi_{1}(M)$ is negatively curved we proved that $\mathcal{B}_{L}$ is always finite, irrespective of $\Phi$ being quasigeodesic or not. The notation will be $\mathcal{B}_{L}=\left\{L_{i}\right\}, 1 \leq i \leq n$.

Our goal is to show that if $\Phi$ is quasigeodesic and $\mathcal{B}_{L}$ is the total limit of a monotone convergent sequence $F_{i} \in \widetilde{\mathcal{F}}^{s}$ as $i \rightarrow \infty$, then $\bigcup_{j=1}^{n} \Lambda_{L_{j}}$ is the limit of $\Lambda_{F_{i}}$ in the Hausdorff topology.

Theorem 4.2. Let $\Phi$ be an Anosov flow in $M^{3}$ with negatively curved $\pi_{1}(M)$. Suppose that $\Phi$ has the continuous extension property. Let $\left\{F_{i}\right\}$ be a monotone, convergent sequence in $\widetilde{\mathcal{F}}^{s}$ and let $\bigcup_{1 \leq j \leq n}\left\{L_{j}\right\}$ be its total limit. Suppose that $\Lambda_{F_{i}}$ converges in the Hausdorff topology. Then

$$
\bigcup_{1 \leq j \leq n} \Lambda_{L_{j}} \subset \lim _{i \rightarrow \infty} \Lambda_{F_{i}} .
$$

Proof. Let $p \in \bigcup_{1 \leq j \leq n} \Lambda_{L_{j}}$. Without loss of generality assume that $p \in \Lambda_{L_{1}}$ and let $L=L_{1}$. Assume also that the $F_{i}$ are all in back of $L$. 
Assume first that $p=\eta_{-}(x)$ for some $x \in L$. Then $\widetilde{W}_{-}^{u u}(x)$ intersects $F_{i}$ for all $i$ big enough. Let $y_{i}=\widetilde{W}_{-}^{u u}(x) \cap F_{i}$. Since $x \in \widetilde{W}^{u}\left(y_{i}\right)$, then $\eta_{-}(x)=\eta_{-}\left(y_{i}\right) \in \Lambda_{F_{i}}$ for all $i$ big enough, so in fact $p \in \Lambda_{F_{i}}$ for all $i$ big enough, and clearly $p$ is in the limit of $\Lambda_{F_{i}}$ in the Hausdorff topology.

If on the other hand $p=\eta_{+}(L)$, then let $z \in L$, so $p=\eta_{+}(z)$. For big $i$, let $y_{i}=\widetilde{W}_{-}^{u u}(z) \cap F_{i}$. Then $y_{i} \rightarrow z$ in $\widetilde{M}$, so $\eta_{+}(z)=\lim _{i \rightarrow \infty} \eta_{+}\left(y_{i}\right)$. So clearly $z \in \lim _{i \rightarrow \infty} \Lambda_{F_{i}}$.

This was an easy fact. In general limit sets could explode in the limit, so to get the converse subset relation, we will need to have better control of where flow lines roughly are. In order to achieve that we will assume quasigeodesic behavior.

Theorem 4.3. Let $\Phi$ be a quasigeodesic Anosov flow in $M^{3}$ with $\pi_{1}(M)$ negatively curved. Let $F_{i}$ be a monotone, convergent sequence in $\widetilde{\mathcal{F}}^{s}$ and let $\bigcup_{1 \leq j \leq n} L_{j}$ be its total limit. Then $\Lambda_{F_{i}}$ converges in the Hausdorff topology, and

$$
\bigcup_{1 \leq j \leq n} \Lambda_{L_{j}}=\lim _{i \rightarrow \infty} \Lambda_{F_{i}}
$$

Proof. Let $A=\bigcup_{1 \leq j \leq n} \Lambda_{L_{j}}$ and $L=L_{1}$. Let $z \in A$. The proof of the previous theorem shows that there is a sequence $y_{i} \in \Lambda_{F_{i}}$ with $y_{i} \rightarrow z$.

Conversely, let $q_{n}$ in the limit set of $F_{i_{n}}$ with $q_{n} \rightarrow q \in S_{\infty}^{2}$ and $i_{n} \rightarrow \infty$. We want to show that $q \in A$. If $q_{n}=\eta_{+}\left(F_{i_{n}}\right)$ for infinitely many $n$, then as $\eta_{+}\left(F_{i_{n}}\right) \rightarrow \eta_{+}(L)$ we would get $q \in \Lambda_{L} \subset A$.

So we assume that $q_{n}=\eta_{-}\left(x_{n}\right)$, where $x_{n} \in F_{i_{n}}$. If $q_{n} \rightarrow \eta_{+}(L)$, we are done. So assume $q \neq \eta_{+}(L)$. We will use the following facts about spaces which are negatively curved in the large [Gr], [Gh-Ha]:

(1) There is an $\epsilon_{1}>0$ so that for any $u \neq v \in S_{\infty}^{2}$ and any two minimal geodesics $\alpha_{1}, \alpha_{2}$ with ideal points $u, v$ it must be true that $\alpha_{1}$ and $\alpha_{2}$ are at most $\epsilon_{1}$ apart (that is, $\alpha_{1} \subset N\left(\alpha_{2}, \epsilon_{1}\right)$ and $\alpha_{2} \subset N\left(\alpha_{1}, \epsilon_{1}\right)$ ).

(2) Let $u \neq v \in S_{\infty}^{2}$ and $u_{i}, v_{i} \in \widetilde{M} \cup S_{\infty}^{2}$ with $u_{i} \rightarrow u, v_{i} \rightarrow v$. If the $\gamma_{i}$ are minimal geodesics (or minimal geodesic segments or minimal geodesic rays) connecting $u_{i}$ and $v_{i}$, then there is a subsequence $\gamma_{i_{m}}$ converging (in the Hausdorff topology of $\widetilde{M} \cup S_{\infty}^{2}$ ) to a minimal geodesic $\gamma$ connecting $u$ and $v$.

(3) These two facts imply the following: Let $x$ be a point in a minimal geodesic with endpoints $u, v \in S_{\infty}^{2}$. For any $\epsilon_{2}>0$ there are disjoint neighborhoods $U, V$ of $u, v$ in $\widetilde{M} \cup S_{\infty}^{2}$ so that any minimal geodesic $\alpha$ with endpoints in $u^{\prime} \in U$ and $v^{\prime} \in V$ intersects $N\left(x, \epsilon_{1}+\epsilon_{2}\right)$.

So let $\gamma$ be any minimal geodesic of $\widetilde{M}$ with endpoints $\eta_{+}(L)$ and $q$. Choose $x \in \gamma$. Suppose that $\gamma_{n}$ are minimal geodesics of $\widetilde{M}$ with endpoints $\eta_{+}\left(x_{n}\right)$ and $\eta_{-}\left(x_{n}\right)=q_{n}$. As $\Phi$ is $k$-quasigeodesic, it follows that $\widetilde{\Phi}_{\mathbf{R}}\left(x_{n}\right) \subset N\left(\gamma_{n}, R\right)$ for all $n$. Since $\eta_{+}\left(x_{n}\right) \rightarrow \eta_{+}(L)$ and $\eta_{-}\left(x_{n}\right)=q_{n} \rightarrow q \neq \eta_{+}(L)$, fact (3) above implies that for big enough $n, \gamma_{n}$ intersects $N\left(x, \epsilon_{1}+\epsilon_{2}\right)$. Therefore $\widetilde{\Phi}_{\mathbf{R}}\left(x_{n}\right)$ intersects $N\left(x, \epsilon_{1}+\epsilon_{2}+R\right)$. Let $y_{n}$ be a point in this intersection, and up to a further subsequence assume that $y_{n} \rightarrow y \in \widetilde{M}$. As $\eta_{-}$is a continuous function in $\widetilde{M}$, it follows that $\eta_{-}\left(y_{n}\right) \rightarrow \eta_{-}(y)$. But $\eta_{-}\left(y_{n}\right)=\eta_{-}\left(x_{n}\right)=q_{n}$. So $q=\eta_{-}(y)$.

On the other hand, $y_{n} \in F_{i_{n}}$, and $y_{n} \rightarrow y$ implies that $y \in E \in \widetilde{\mathcal{F}}^{s}$, so $E \in$ $\mathcal{T} L\left(F_{i_{n}}\right)$. Therefore $y \in L_{j}$ for some $1 \leq j \leq n$. This implies that $q=\eta_{-}(y) \in \Lambda_{L_{j}}$. 
(a)

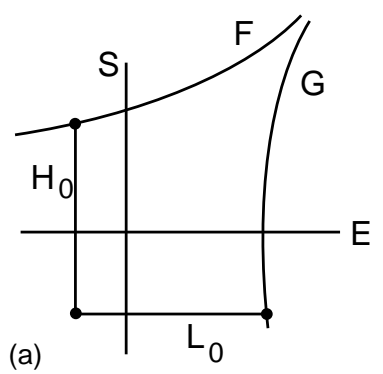

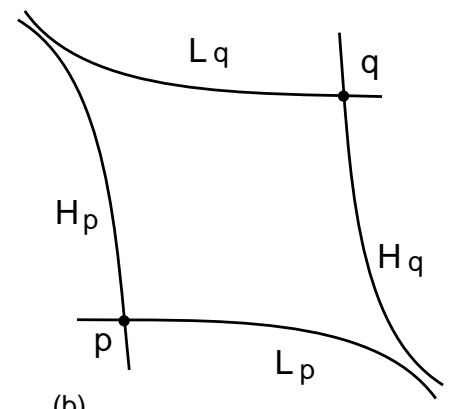

(b)

Figure 2. (a) Perfect fits in the universal cover. (b) Lozenge. proof.

We conclude that $\lim \Lambda_{F_{i}}$ exists and is equal to $\bigcup_{1 \leq j \leq n} \Lambda_{L_{j}}$. This finishes the

\section{INTERSECTION OF LIMIT SETS}

Suppose that $\Phi$ is quasigeodesic. In this section we completely determine when $\Lambda_{F} \cap \Lambda_{L} \neq \emptyset$, for leaves $F, L \in \widetilde{\mathcal{F}}^{s}$ or $\widetilde{\mathcal{F}}^{u}$. Since $\Lambda_{F}=\phi_{F}\left(\partial_{\infty} F\right)$ and $\partial_{\infty} F$ consists of intrinsic ideal points of flow lines in $F$, then nontrivial intersection implies that there are flow lines in $F, L$ which share an ideal point in $S_{\infty}^{2}$. The strategy is to use the quasigeodesic property to determine exactly when flow lines share an ideal point. We need a few preliminary definitions:

If $L_{0}$ is a half leaf of $F \in \widetilde{\mathcal{F}}^{s}$ or $\widetilde{\mathcal{F}}^{u}$ defined by the orbit $\gamma$ of $\widetilde{\Phi}$, then its closure is $\bar{L}_{0}=L \cup\{\gamma\}$ and its boundary is $\partial L_{0}=\{\gamma\}$. If $\beta, \gamma$ are two distinct flow lines of $\widetilde{\Phi}$ in a leaf $L$ of $\widetilde{\mathcal{F}}^{s}$ or $\widetilde{\mathcal{F}}^{u}$, then the flow band defined by $\beta, \gamma$ is the connected component $B$ of $L-\{\gamma, \beta\}$ which is not a half leaf of $L$. The closed flow band is $\bar{B}=B \cup\{\beta, \gamma\}$, and its boundary is $\partial B=\{\beta, \gamma\}$.

Definition 5.1 (perfect fits). Two leaves $F \in \widetilde{\mathcal{F}}^{s}, G \in \widetilde{\mathcal{F}}^{u}$ form a perfect fit if $F \cap G=\emptyset$ and there are open half leaves $F_{0}$ of $F$ and $G_{0}$ of $G$ and flow bands $L_{0} \subset L \in \widetilde{\mathcal{F}}^{s}$ and $H_{0} \subset H \in \widetilde{\mathcal{F}}^{u}$ so that

$$
\begin{gathered}
\bar{L}_{0} \cap \bar{G}_{0}=\partial L_{0} \cap \partial G_{0}, \bar{L}_{0} \cap \bar{H}_{0}=\partial L_{0} \cap \partial H_{0}, \bar{H}_{0} \cap \bar{F}_{0}=\partial H_{0} \cap \partial F_{0}, \\
\forall S \in \widetilde{\mathcal{F}}^{u} \quad S \cap L_{0} \neq \emptyset \Leftrightarrow S \cap F_{0} \neq \emptyset
\end{gathered}
$$

and

$$
\forall E \in \widetilde{\mathcal{F}}^{s} \quad E \cap G_{0} \neq \emptyset \Leftrightarrow E \cap H_{0} \neq \emptyset .
$$

We also say that $F$ and $G$ are asymptotic in the sense that leaves of $\widetilde{\mathcal{F}}^{s}$ near $F$ and on the side that $G$ is on, will intersect $G$, and vice versa.

Definition 5.2 (Lozenges). Let $p, q \in \widetilde{M}, p \notin \widetilde{W}^{s}(q), p \notin \widetilde{W}^{u}(q)$. Let $H_{p}$ be the half leaf of $\widetilde{W}^{u}(p)$ defined by $\widetilde{\Phi}_{\mathbf{R}}(p)$ and contained in the same side of $\widetilde{W}^{s}(p)$ as $q$. Similarly define $H_{q}$. Also define $L_{p}$ to be the half leaf of $\widetilde{W}^{s}(p)$ defined by $\widetilde{\Phi}_{\mathbf{R}}(p)$ on the same side of $\widetilde{W}^{u}(p)$ as $q$. Similarly define $L_{q}$. Then $p, q$ form a lozenge, fig. 2(b) if $H_{p}, L_{q}$ and $H_{q}, L_{p}$ respectively form perfect fits. 


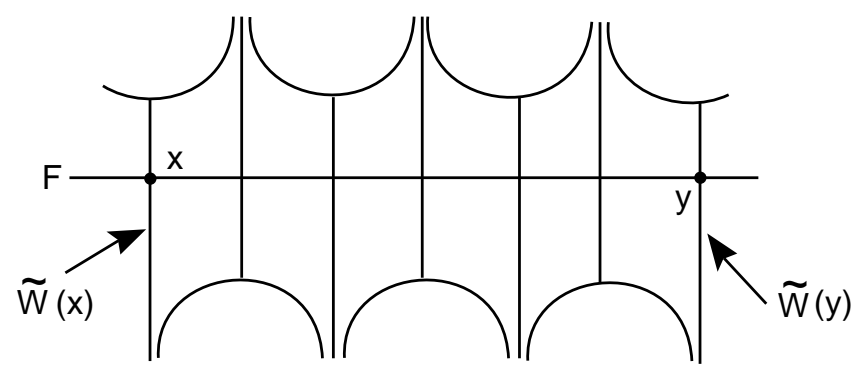

FiguRE 3. Identification of ideal points in a stable leaf generates a chain of lozenges connecting unstable leaves.

We say that $p, q$ are corners of the lozenge. Two lozenges are adjacent if they share a corner and there is a stable (or unstable) leaf intersecting both interiors. A sequence of lozenges $Z_{i}$ forms a chain if each $Z_{i}$ and $Z_{i+1}$ share a corner. Consecutive lozenges may be adjacent or not.

Theorem 5.3 ([Fe8]). Let $\Phi$ be a non-R-covered Anosov flow in $M^{3}$, closed. Then if $F, L$ is a pair of branching leaves of $\widetilde{\mathcal{F}}^{s}$, there is a nontrivial covering translation $g$ leaving both of them invariant. Hence $F, L$ are periodic. In addition, the periodic orbits in $F, L$ are connected by a chain of adjacent lozenges all of which intersect a common stable leaf. There are an even number of lozenges in the chain.

The following result specifies exactly when different flow lines in the same stable (or unstable) leaf have the same ideal points. It is the fundamental tool used in this section.

Theorem 5.4 ([Fe4]). Let $\Phi$ be an Anosov flow in $M^{3}$ with negatively curved $\pi_{1}(M)$. Suppose that $\Phi$ is quasigeodesic. Let $x, y \in F \in \widetilde{\mathcal{F}}^{s}$ be not in the same flow line of $\widetilde{\Phi}$ and satisfy $\eta_{-}(x)=\eta_{-}(y)$. Then $\widetilde{W}^{u}(x), \widetilde{W}^{u}(y)$ (which are not the same leaves) are periodic, fixed by a common covering translation $g$, and the periodic orbits in $\widetilde{W}^{u}(x), \widetilde{W}^{u}(y)$ are connected by a chain of adjacent lozenges, all intersecting $F$. There are an even number of lozenges in the chain; see fig. 3. Analogous results hold if $\eta_{+}(x)=\eta_{+}(y)$ and $x, y \in G \in \widetilde{\mathcal{F}}^{u}$.

Lemma 5.5. If $F, L, G \in \widetilde{\mathcal{F}}^{s}$ (or $\widetilde{\mathcal{F}}^{u}$ ), $p \in \Lambda_{F} \cap \Lambda_{L}$ and $G$ separates $F$ from $L$ in $\widetilde{M}$, then $p \in \Lambda_{G}$.

Proof. If $p \notin \Lambda_{G}$, let $U^{\prime}$ be a neighborhood of $p \in \widetilde{M} \cup S_{\infty}^{2}$ disjoint from $G$. Since $p \in \Lambda_{F} \cap \Lambda_{G}$, there are $x \in F \cap U$ and $y \in L \cap U$. Then $x$ can be connected to $y$ in $U$ and hence $G$ does not separate $F$ from $L$, a contradiction.

Lemma 5.6. Let $\Phi$ be an Anosov flow in $M^{3}$ with $\pi_{1}(M)$ negatively curved and so that $\Phi$ has the continuous extension property. If $F, L$ are nonseparated leaves in $\widetilde{\mathcal{F}}^{s}$, then $\eta_{+}(F)=\eta_{+}(L)$. Similarly for $\widetilde{\mathcal{F}}^{u}$. In addition, if $F \in \widetilde{\mathcal{F}}^{s}$ and $G \in \widetilde{\mathcal{F}}^{u}$ form a perfect fit, then $\eta_{+}(F)=\eta_{-}(G)$.

Proof. The first statement follows from the proof of proposition 4.9 of [Fe4]. For the second part let $A=\widetilde{\Phi}_{\mathbf{R}}\left(\zeta_{1}\right)$, where $\zeta_{1}$ is a strong stable segment, $B=\widetilde{\Phi}_{\mathbf{R}}\left(\zeta_{2}\right)$, where $\zeta_{2}$ is a strong unstable segment; and let $F_{0}, G_{0}$ be half leaves of $F$ and $G$ so that $A, B, F_{0}, G_{0}$ satisfy the requirements of perfect fits. 
Let $x=\partial \zeta_{2} \cap F$ and let $x_{i} \in \zeta_{2}$ with $x_{i} \rightarrow x$. Hence $\emptyset \neq \widetilde{W}^{s}\left(x_{i}\right) \cap G_{0}=\gamma_{i}$. Then $\gamma_{i}$ is a collection of orbits of $\widetilde{\Phi}$ in $G$. If $\gamma_{i}$ does not escape in $G$, this limit is on an orbit $\gamma$ of $\widetilde{\Phi}$ in $G$. Consequently $\widetilde{W}^{s}(\gamma)$ and $F$ are not separated in $\widetilde{\mathcal{F}}^{s}$, hence they do not intersect a common transversal to $\widetilde{\mathcal{F}}^{s}$. Notice that $F \neq \widetilde{W}^{s}(\gamma)$. Since $\gamma \subset G$, for $y \in \zeta_{1}$ near enough $\partial \zeta_{1} \cap G$ we have $\widetilde{W}^{u}(y) \cap \widetilde{W}^{s}(\gamma) \neq \emptyset$; hence $\widetilde{W}^{u}(y) \cap F=\emptyset$. This contradicts the fact that for any $y^{\prime} \in \zeta_{1}, \widetilde{W}^{u}\left(y^{\prime}\right) \cap F_{0} \neq \emptyset$.

Therefore the intrinsic positive limit points of $\gamma_{i}$ in $\partial_{\infty} G$ converge to the intrinsic negative limit point of $G$ in $\partial_{\infty} G$. Since the map $\phi: G \rightarrow \widetilde{M}$ extends continuously to $\phi: G \cup \partial_{\infty} G \rightarrow \widetilde{M} \cup S_{\infty}^{2}$, then $\eta_{+}\left(\gamma_{i}\right) \rightarrow \eta_{-}(G)$.

On the other hand, since $\eta_{+}$is a continuous function then $\eta_{+}(x)=\lim \eta_{+}\left(x_{i}\right)$ as $i \rightarrow \infty$. As $\gamma_{i} \subset \widetilde{W}^{s}\left(x_{i}\right)$, then $\eta_{+}\left(\gamma_{i}\right)=\eta_{+}\left(x_{i}\right)$. We conclude that $\eta_{+}(F)=$ $\eta_{+}(x)=\eta_{-}(G)$. This finishes the proof.

This result shows that whenever $S, R \in \widetilde{\mathcal{F}}^{s}$ or $\widetilde{\mathcal{F}}^{s}$ are either nonseparated or form a perfect fit, they share a limit point in $S_{\infty}^{2}$. The same is true for sequences of leaves such that consecutive leaves satisfy one of the above conditions. The main result of this section states that when in addition $\Phi$ is quasigeodesic, this is the only possibility for limit sets to intersect.

Theorem 5.7. Let $\Phi$ be a quasigeodesic Anosov flow in $M^{3}$ with negatively curved fundamental group. Let $x, y \in \widetilde{M}$, not in the same orbit of $\widetilde{\Phi}$ be such that $\eta_{+}(x)=$ $\eta_{+}(y)$. Let $F=\widetilde{W}^{s}(x), L=\widetilde{W}^{s}(y)$. Then one of the following mutually exclusive alternatives happens:

(1) $F=L$.

(2) $F \neq L$ but they intersect a common unstable leaf $G$. In this case, $F, L$ are periodic, invariant under a nontrivial covering translation $g$, and the periodic orbits in $F, L$ are connected by an even chain of adjacent lozenges all intersecting $G$. Notice that $x, y$ may be in $G$.

(3) $F \neq L$ and $F, L$ do not intersect a common unstable leaf. Then there is a sequence of leaves $S_{i}, 0 \leq i \leq m$, with $S_{i} \in \widetilde{\mathcal{F}}^{s}$ or $\widetilde{\mathcal{F}}^{u}$, such that $S_{0}=F, S_{m}=L$ and for each $0 \leq i<m$, either $S_{i}$ and $S_{i+1}$ form a perfect fit, or $S_{i}, S_{i+1}$ are in the same foliation (stable or unstable), in which case they are nonseparated leaves in this foliation.

In general if any leaf $S_{i}$ is periodic, then all leaves in the collection are periodic and invariant under a common nontrivial covering translation. Then the periodic orbits in $F, L$ are connected by a chain of lozenges, not necessarily all adjacent. In particular, this happens if there is any branching in the sequence.

Proof. Case (1) is obvious since all flow lines in $F$ are forward asymptotic. In case (2) let $z \in F \cap G$ and let $w \in L \cap G$. Then $\eta_{+}(z)=\eta_{+}(x)=\eta_{+}(y)=\eta_{+}(w)$ and $\widetilde{W}^{u}(z)=\widetilde{W}^{u}(w)$. The result now follows from theorem 5.4.

Suppose then that $F$ and $L$ do not intersect a common unstable leaf. Let $p=$ $\eta_{+}(x)=\eta_{+}(y)$. Let

$$
\mathcal{C}=\left\{G \in \widetilde{\mathcal{F}}^{u} \mid G \cap F \neq \emptyset\right\} .
$$

Then $\mathcal{C}$ is an open, connected, $\widetilde{\mathcal{F}}^{u}$ saturated set and $L \cap \mathcal{C}=\emptyset$. Since $\partial \mathcal{C}$ is a union of unstable leaves, it follows that $L \cap \partial \mathcal{C}=\emptyset$ also. Hence there is a unique unstable leaf $H_{1} \subset \partial \mathcal{C}$ separating $\mathcal{C}$ from $L$; see fig. 4(a). In particular, $H_{1} \cap L=\emptyset$. Parametrize 

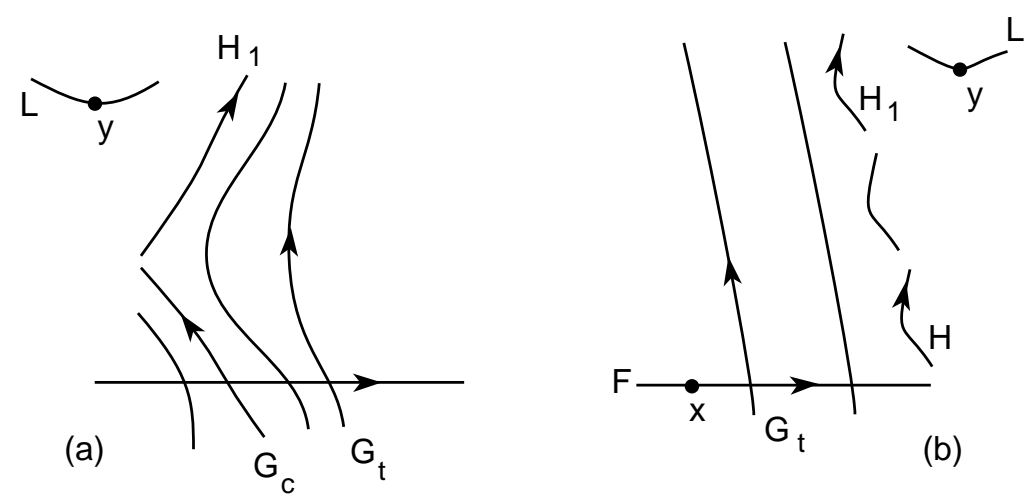

Figure 4. Generating intersection of limits sets of leaves.

the leaves in $\mathcal{C}$ by $\left\{G_{t} \mid t \in \mathbf{R}\right\}$, where $t$ increases in the positive direction transverse to $\widetilde{\mathcal{F}}^{u}$. Since leaves of $\widetilde{\mathcal{F}}^{u}$ separate $\widetilde{M}$, there are two possibilities:

(I) There is $c \in \mathbf{R}$ so that $L$ is in front of $G_{t}$ for $t<c$ and $L$ is in back of $G_{t}$ for $t>c$.

If $H_{1}$ is separated from $G_{c}$, then there exist $\epsilon>0$ and an $\widetilde{\mathcal{F}}^{u}$ saturated neighborhood $\mathcal{U}$ of $H_{1}$ so that $\mathcal{U}$ does not contain any $G_{t}$ for $|t-c|<\epsilon$. Since $L$ is in back of $G_{c+\epsilon / 2}$ and $H_{1}$ separates $L$ from $\mathcal{C}$, then $H_{1}$ is also in back of $G_{c+\epsilon / 2}$. Similarly, as $L$ is in front of $G_{c-\epsilon / 2}$, then $H_{1}$ is in front of $G_{c-\epsilon / 2}$. Since $H_{1}$ is in the boundary of $\mathcal{C}$, this implies that $\mathcal{U}$ has to contain some $G_{t}$ with $|t-c|<\epsilon / 2$, a contradiction. Hence $H_{1}$ and $G_{c}$ are not separated.

Without loss of generality assume that $L$ is in front of $G_{c}$ the other case being similar. Then $H_{1}$ is in the limit of $G_{t}, t \rightarrow c, t>c$. Furthermore $H_{1}$ and $G_{c}$ are not separated on their positive sides; see fig. 4(a).

As $H_{1}$ separates $L$ from $\mathcal{C}$, it also separates $L$ from $F$. Since $p \in \Lambda_{F} \cap \Lambda_{L}$, by lemma 5.5, $p \in \Lambda_{H_{1}}$. So $p$ is either $\eta_{-}\left(H_{1}\right)$ or $\eta_{+}(z)$ for some $z \in H_{1}$. In the first case, since $H_{1}$ is not separated from $G_{c}$, then by lemma 5.6, $\eta_{-}\left(H_{1}\right)=\eta_{-}\left(G_{c}\right)=p$. Let $x^{\prime} \in G_{c} \cap F$. Since $x^{\prime} \in \widetilde{W}^{s}(x), \eta_{+}\left(x^{\prime}\right)=\eta_{+}(x)=p$; and since $x^{\prime} \in G_{c}$, $\eta_{-}\left(x^{\prime}\right)=\eta_{-}\left(G_{c}\right)=p$. This contradicts the fact that $\widetilde{\Phi}_{\mathbf{R}}\left(x^{\prime}\right)$ is a quasigeodesic.

Therefore $p=\eta_{+}\left(z_{1}\right)$ for some $z_{1} \in H_{1}$. But then $\eta_{+}\left(\widetilde{W^{s}}\left(z_{1}\right)\right)=\eta_{+}(F)$. Since $H_{1}$ and $G_{c}$ are not separated on their positive sides and $\widetilde{W}^{s}\left(z_{1}\right) \cap H_{1} \neq \emptyset, F \cap G_{c} \neq \emptyset$, it follows that $F$ and $\widetilde{W}^{s}\left(z_{1}\right)$ intersect a common unstable leaf. We can then apply case (2) of the theorem and deduce that $F, \widetilde{W}^{s}\left(z_{1}\right)$ are periodic and invariant under a common nontrivial covering translation $g$.

Let $\gamma_{0}$ the periodic orbit in $F$ and $\gamma_{1}$ be the periodic orbit in $\widetilde{W}^{s}\left(z_{1}\right)$. By theorem 5.4, $\gamma_{0}$ and $\gamma_{1}$ are connected by a chain of adjacent lozenges $B_{i}, 1 \leq i \leq m_{0}$, all intersecting a common unstable leaf. Since $G_{c}$ and $H_{1}$ form a branching pair of $\widetilde{\mathcal{F}}^{u}$ and $\widetilde{W}^{s}\left(\gamma_{0}\right) \cap G_{c} \neq \emptyset, \widetilde{W}^{s}\left(\gamma_{1}\right) \cap H_{1} \neq \emptyset$, the proof of theorem 4.11 of [Fe4] shows that in fact $\gamma_{0} \subset G_{c}$ and $\gamma_{1} \subset H_{1}$. Therefore $\widetilde{W}^{s}\left(z_{1}\right) \cap H_{1}=\gamma_{1}=\widetilde{\Phi}_{\mathbf{R}}\left(z_{1}\right)$, so $z_{1}$ is in the periodic orbit in $H_{1}$. Notice that $F$ forms a perfect fit with a leaf $Y_{0} \in \widetilde{\mathcal{F}}^{u}$ which is in the boundary of the chain of lozenges. Furthermore, $\widetilde{W}^{s}\left(z_{1}\right)$ forms a perfect fit with a leaf $Y_{1}$ which is not separated from $Y_{0}\left(Y_{1}\right.$ may be equal to $\left.Y_{0}\right)$. The leaves $Y_{0}, Y_{1}$ are in the boundary of the chain of lozenges. 


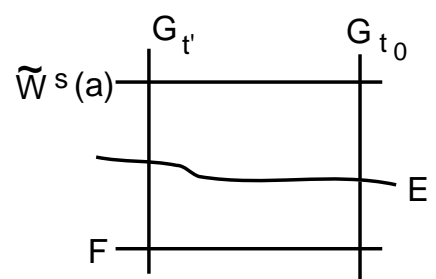

(a)

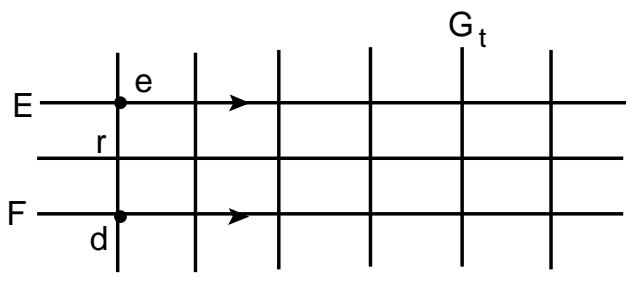

(b)

Figure 5. (a) A rectangle in the universal cover, (b) Product region.

In this case $\eta_{+}\left(z_{1}\right)=\eta_{+}(x)=\eta_{+}(y)$. We can then restart the whole analysis with $z_{1}$ and $y$.

(II) Either for all $t$ the leaf $L$ is in front of $G_{t}$, or for all $t, L$ is in back of $G_{t}$.

Without loss of generality assume the first option, that is, $\mathcal{C}$ is contained in back of $L$. Then $H_{1}$ is in the front of all $G_{t}$ and as a result $G_{t} \rightarrow H_{1}$ as $t \rightarrow+\infty$ (and maybe $G_{t}$ converges to other leaves of $\widetilde{\mathcal{F}}^{u}$ too); see fig. 4(b). Then $\eta_{-}\left(G_{t}\right) \rightarrow$ $\eta_{-}\left(H_{1}\right)$. But $\eta_{-}\left(G_{t}\right)=\eta_{-}\left(\alpha_{t}\right)$, where $\alpha_{t}=G_{t} \cap F$. Since $\alpha_{t}$ escapes to infinity in $F$, then $\eta_{-}\left(\alpha_{t}\right) \rightarrow \eta_{+}(F)$. We conclude that $\eta_{+}(F)=\eta_{-}\left(H_{1}\right)$, so $\eta_{-}\left(H_{1}\right)=p$. Notice that this implies that for any $u \in H_{1}$ we have $\eta_{+}(u) \neq p=\eta_{+}(x)$. We caution the reader that in general $H_{1}$ and $F$ do not form a perfect fit.

If $G_{t}$ has limits on more than one leaf of $\widetilde{\mathcal{F}}^{u}$, then it produces branching in $\widetilde{\mathcal{F}}^{u}$ and $H_{1}$ will be periodic. In any case, since $\pi_{1}(M)$ is negatively curved, there are only finitely many leaves in the set $\mathcal{B}_{H_{1}}$ of leaves of $\widetilde{\mathcal{F}}^{u}$ not separated from $H_{1}$ and in back of $H_{1}$. Put the following order in $\mathcal{B}_{H_{1}}$ : given $U, U^{\prime} \in \mathcal{B}_{H_{1}}$, choose $E, E^{\prime} \in \widetilde{\mathcal{F}}^{s}$ with $E \cap U \neq \emptyset, E^{\prime} \cap U^{\prime} \neq \emptyset$. Then define $U<U^{\prime}$ if $E$ is in back of $E^{\prime}$. Assume that $L$ is in front of $F$, hence $H_{1}$ is in front of $F$. Let $H$ be the smallest element of $\mathcal{B}_{H_{1}}$ which is in front of $F$.

Lemma 5.8. $F$ and $H$ form a perfect fit.

Proof. Let $a \in H$ and let $\zeta_{1}$ be a small open segment in $\widetilde{W}_{-}^{s s}(a)$ with one endpoint in $a$. Since $G_{t} \rightarrow H$ as $t \rightarrow+\infty$, choose $\zeta_{1}$ small enough so that it only intersects unstable leaves which are in $\mathcal{C}$. Let $b$ be the other endpoint of $\zeta_{1}$. Then $b \in G_{t^{\prime}}$ for some $t^{\prime} \in \mathbf{R}$. Let $A=\widetilde{\Phi}_{\mathbf{R}}\left(\zeta_{1}\right)$, which is a flow band in $\widetilde{W}^{s}(a)$. Let $d=\widetilde{W}_{-}^{u u}(b) \cap F$, and let $\zeta_{2}$ be the open segment in $\widetilde{W}^{u u}(b)$ with endpoints $b, d$. Let $B=\widetilde{\Phi}_{\mathbf{R}}\left(\zeta_{2}\right)$, a flow band in $\widetilde{W}^{u}(b)$. Let $F^{\prime}=\widetilde{W}_{+}^{s}(d)$ and $H^{\prime}=\widetilde{W}_{-}^{u}(a)$. We will show that $A, B, F^{\prime}$ and $H^{\prime}$ satisfy the requirements of a perfect fit. By construction

$$
\left\{Y \in \widetilde{\mathcal{F}}^{u} \mid Y \cap A \neq \emptyset\right\}=\left\{G_{t} \mid t>t^{\prime}\right\}=\left\{Y \in \widetilde{\mathcal{F}}^{u} \mid Y \cap F^{\prime} \neq \emptyset\right\} .
$$

Now let $E \in \widetilde{\mathcal{F}}^{s}$ with $E \cap H^{\prime} \neq \emptyset$. Since $G_{t} \rightarrow H_{1}$ as $t \rightarrow \infty$, then for all $t$ big enough $E \cap G_{t} \neq \emptyset$ and $\widetilde{W}^{s}(a) \cap G_{t} \neq \emptyset$. Choose one such $t_{0}$. Notice that $F \cap G_{t_{0}} \neq \emptyset, F \cap G_{t^{\prime}} \neq \emptyset, \widetilde{W}^{s}(a) \cap G_{t_{0}} \neq \emptyset$ and $\widetilde{W}^{s}(a) \cap G_{t^{\prime}} \neq \emptyset$. Therefore $F, G_{t_{0}}, \widetilde{W}^{s}(a)$ and $G_{t^{\prime}}$ form a rectangle in $\widetilde{M}$; see fig. 5 (a). Since $E$ intersects $G_{t_{0}}$ between $\widetilde{W}^{s}(a)$ and $F$, then by lemma 4.1 of [Fe7] it also intersects $G_{t^{\prime}}$ between $\widetilde{W}^{s}(a)$ and $F$. But the subset of the leaf $G_{t^{\prime}}$ which is between $\widetilde{W^{s}}(a) \cap G_{t^{\prime}}$ and $F \cap G_{t^{\prime}}$ is exactly $B$; hence $E \cap B \neq \emptyset$. 
Conversely, let $E \in \widetilde{\mathcal{F}}^{s}$ with $E \cap B \neq \emptyset$. Suppose that $E \cap H^{\prime}=\emptyset$. Let $e=E \cap \zeta_{2}$, and let $\beta$ be the closed segment in $\widetilde{W}^{u u}(b)$ with endpoints $e$ and $d$. Let $r \in \beta$.

For any $t>t^{\prime}, G_{t} \cap \widetilde{W}^{s}(a) \neq \emptyset$ and $G_{t} \cap F \neq \emptyset$. Since $\widetilde{W}^{s}(r)$ separates $F$ from $\widetilde{W}^{s}(a)$, then $G_{t} \cap \widetilde{W}_{+}^{s}(r) \neq \emptyset$. Consider $r_{t}=G_{t} \cap \widetilde{W}_{+}^{s s}(r)$ for $t>t^{\prime}$. If $r_{t}$ does not escape to infinity in $\widetilde{W}_{+}^{s s}(r)$, then $r_{t} \rightarrow r^{\prime} \in \widetilde{W}_{+}^{s s}(r)$ when $t \rightarrow+\infty$. This would imply that $\widetilde{W}^{u}\left(r^{\prime}\right)$ is not separated from $H_{1}$; hence $\widetilde{W}^{u}\left(r^{\prime}\right) \in \mathcal{B}_{H_{1}}$. In addition, $\widetilde{W}^{u}\left(r^{\prime}\right)<H$ in the order of $\mathcal{B}_{H_{1}}$, because $\widetilde{W}^{s}(a)$ is in front of $\widetilde{W}^{s}(r)$. Since $\widetilde{W}^{u}\left(r^{\prime}\right)$ does not intersect $F$, as it is not $G_{t}$ for any $t \in \mathbf{R}$, then $\widetilde{W}^{u}\left(r^{\prime}\right)$ is in front of $F$. This is a contradiction to the definition of $H$. Hence $r_{t} \rightarrow \infty$ in $\widetilde{W}_{+}^{s s}(r)$.

This implies that if $Y \in \widetilde{\mathcal{F}}^{u}$ and $Y \cap \widetilde{W}_{+}^{s}(r) \neq \emptyset$, then $Y=G_{t}$ for some $t>t^{\prime}$. Hence the set

$$
\left\{Y \in \widetilde{\mathcal{F}}^{u} \mid Y \cap \widetilde{W}_{+}^{s}(r) \neq \emptyset\right\}
$$

is independent of $r \in \beta$. Therefore $\beta$ is the basis segment of a stable product region in $\widetilde{M}$ (detailed definition in section 5 of [Fe8]); see fig. 5 (b). Theorem 5.1 of [Fe8] shows that the existence of a product region in $\widetilde{M}$ implies that $\Phi$ is $\mathbf{R}$-covered, which is a contradiction. Therefore $E \cap B \neq \emptyset \Leftrightarrow E \cap H^{\prime} \neq \emptyset$.

We conclude that $H$ and $F$ form a perfect fit.

Continuation of the proof of theorem 5.7.

If $\mathcal{B}_{H_{1}} \neq H_{1}$, then all leaves in $\mathcal{B}_{H_{1}}$ are periodic. In that case, since $H$ is periodic and $F, H$ form a perfect fit, then by lemma 4.3 of [Fe6], $F$ is also periodic and invariant under the same covering translations that leave $H$ invariant.

In any case $F$ makes a perfect fit with $H$ and $H$ is not separated from $H_{1}$. Therefore $\eta_{+}(F)=\eta_{-}(H)=\eta_{-}\left(H_{1}\right)=\eta_{+}(L)=\eta_{+}(y)$. Choose $z_{1} \in H_{1}$; then $\eta_{-}\left(z_{1}\right)=\eta_{+}(y)$. Notice that $H_{1} \cap L=\emptyset$. Let

$$
\mathcal{C}_{1}=\left\{E \in \widetilde{\mathcal{F}}^{s} \mid E \cap H_{1} \neq \emptyset\right\} .
$$

Then $L \notin \mathcal{C}_{1}$. As before, there is a unique $L_{2} \subset \partial \mathcal{C}_{1}$ which either is $L$ or separates $\mathcal{C}_{1}$ from $L$. Using a similar argument as above, we find $z_{2} \in L_{2}$, so that either $\eta_{-}\left(z_{2}\right)=\eta_{-}\left(z_{1}\right)$ or $\eta_{+}\left(z_{2}\right)=\eta_{-}\left(z_{1}\right)$. The proof then breaks down into two cases analogous to (I) and (II) above.

In this way we use induction to produce a sequence $z_{i}$ so that all flow lines $\widetilde{\Phi}_{\mathbf{R}}\left(z_{i}\right)$ have an ideal point in common. Notice that if $S_{i}$ is an unstable leaf (that is, $S_{i}=\widetilde{W}^{u}\left(z_{i}\right)$ ), then $\eta_{-}\left(z_{i}\right)=\eta_{+}\left(z_{0}\right)$, as opposed to $\eta_{+}\left(z_{i}\right)=\eta_{+}\left(z_{0}\right)$. In addition there are finitely many leaves not separated from any given leaf of $\widetilde{\mathcal{F}}^{s}$ or $\widetilde{\mathcal{F}}^{u}$ (because $\pi_{1}(M)$ is negatively curved). Suppose now that $y$ is never achieved, that is, $\widetilde{W}^{s}\left(z_{i}\right) \neq L$ for all $i$. The above facts imply that there is an infinite subsequence $i_{j}$ so that $\eta_{+}\left(z_{i_{j}}\right)=\eta_{+}(x)$. Let $F_{j}=\widetilde{W}^{s}\left(z_{i_{j}}\right)$. By construction, $F_{j}$ separates $F_{j-1}$ from $F_{j+1}$ for all $j$.

Let $\mathcal{G}_{j}$ be the component of $\widetilde{M}-F_{j}$ which contains $F_{j-1}$. Let $\mathcal{G}=\bigcup_{j \in \mathbf{N}} \mathcal{G}_{j}$. Then $\mathcal{G}$ is open, connected and $\widetilde{\mathcal{F}}^{s}$ saturated. Since $y$ is never achieved, $y \notin \mathcal{G}$. Let $L^{*} \subset \partial \mathcal{G}$, which either is $L$ or separates $L$ from $\mathcal{G}$.

Let $w \in L^{*}$. For $j$ big enough, $\widetilde{W}_{-}^{u}(w)$ intersects $F_{j}$. Furthermore, $\eta_{+}(w)=$ $\lim _{j \rightarrow \infty} \eta_{+}\left(F_{j}\right)$. But $\eta_{+}\left(F_{j}\right)$ is constant, so $\eta_{+}(w)=\eta_{+}\left(x_{i_{j}}\right)$ for all $j$ big enough. Therefore we can apply case (2) of the theorem and conclude that $\widetilde{W}^{s}(w), \widetilde{W}^{s}\left(x_{i_{j}}\right)$ are periodic and the periodic orbits in $\widetilde{W}^{s}(w), \widetilde{W}^{s}\left(z_{i_{j}}\right)$ are connected by a finite 
chain of lozenges. Then $L^{*}$ would be attained through a finite, rather than infinite process, a contradiction.

We conclude that $y$ has to be attained eventually. Hence there is a sequence of points $z_{0}=x, z_{1}, \ldots, z_{n}=y$ so that for every $i$ one of the following possibilities occurs:

(a) $\eta_{+}\left(z_{i-1}\right)=\eta_{+}\left(z_{i}\right)$ and $\widetilde{W}^{s}\left(z_{i-1}\right), \widetilde{W}^{s}\left(z_{i}\right)$ are connected by a chain of adjacent lozenges all intersecting a common unstable leaf;

(b) $\eta_{+}\left(z_{i-1}\right)=\eta_{-}\left(z_{i}\right)$ and there is a leaf $V_{i} \in \widetilde{\mathcal{F}}^{u}$ with $V_{i}$ and $\widetilde{W}^{s}\left(z_{i-1}\right)$ forming a perfect fit and $V_{i}$ not separated from $\widetilde{W}^{u}\left(z_{i}\right)$;

(c) $\eta_{-}\left(z_{i-1}\right)=\eta_{+}\left(z_{i}\right)$, similar to case (b); and

(d) $\eta_{-}\left(z_{i-1}\right)=\eta_{-}\left(z_{i}\right)$, similar to case (a).

This finishes the proof of the theorem.

Remark. The case $\widetilde{W}^{s}(x), \widetilde{W}^{u}(y)$ different but not separated leaves in $\widetilde{\mathcal{F}}^{s}$ is included in alternative (3) even though the algorithm in the proof of the theorem will have an additional step that is not necessary. In the first step the algorithm produces an unstable leaf $G$ making a perfect fit with $\widetilde{W^{s}}(x)$ and separating $\widetilde{W^{s}}(x)$ from $\widetilde{W}^{s}(y)$. It is an unstable leaf in the boundary of the first lozenge of the sequence connecting $\widetilde{W}^{s}(x)$ and $\widetilde{W}^{s}(y)$. In the second step the algorithm produces $\widetilde{W}^{s}(y)$.

The same techniques used in this theorem also tell us when positive and negative limit points are identified.

Corollary 5.9. Let $\Phi$ be a quasigeodesic Anosov flow in $M^{3}$ with $\pi_{1}(M)$ negatively curved. Suppose that $\eta_{-}(x)=\eta_{+}(y)$. Let $F=\widetilde{W}^{s}(y)$ and $G=\widetilde{W}^{u}(x)$. Then case (3) of theorem 5.7 happens.

Proof. First notice that $F \cap G=\emptyset$. Otherwise if $\beta=F \cap G$, then $\eta_{-}(\beta)=\eta_{-}(G)=$ $\eta_{+}(F)=\eta_{+}(\beta)$, a contradiction. Let $p=\eta_{-}(x)$. As in theorem 5.7, let

$$
\mathcal{C}_{1}=\left\{E \in \widetilde{\mathcal{F}}^{s} \mid E \cap G \neq \emptyset\right\} .
$$

Then $F \notin \mathcal{C}_{1}$. As in theorem 5.7, there is a unique stable leaf $L_{1} \subset \partial \mathcal{C}_{1}$ which either is $F$ (in which case we are done) or $L_{1}$ separates $F$ from $\mathcal{C}_{1}$. Then $p \in \Lambda_{L_{1}}$. If $p=\eta_{+}\left(L_{1}\right)$ we can then directly apply theorem 5.7. Otherwise $\eta_{-}(x)=\eta_{-}\left(z_{1}\right)$ for some $z_{1} \in L_{1}$, so we can apply the same procedure to $z_{1}$ and $y$. As shown at the end of the proof of theorem 5.7, this process eventually stops. This finishes the proof.

\section{REFERENCES}

[An] D. V. Anosov, Geodesic flows on closed Riemannian manifolds with negative curvature, Proc. Steklov Inst. Math. 90 (1969). MR 39:3527

[Ba1] T. Barbot, Caractérization des flots d'Anosov en dimension 3 par leurs feuilletages faibles, Erg. Th. Dyn. Sys. 15 (1995) 247-270. MR 96d:58100

[Ba2] T. Barbot, Flots d'Anosov sur les variétés graphées au sens de Waldhausen, Ann. Inst. Fourier (Grenoble) 46 (1996), 1451-1517. MR 97j:57031

[Be-Me] M. Bestvina and J. Mess, The boundary of negatively curved groups, Jour. Amer. Math. Soc. 4 (1991) 469-481. MR 93j:20076

[Bo1] R. Bowen, Periodic orbits for hyperbolic flows, Amer. Jour. of Math. 94 (1972) 1-30. MR 45:7749

[Bo2] R. Bowen, Equilibrium states and the ergodic theory of Anosov diffeomorphims, Lecture Notes in Mathematics 470 Springer Verlag, 1975. MR 56:1374 
[Ca-Th] J. Cannon and W. Thurston, Group invariant Peano curves, to appear.

[Ch] J. Christy, Intransitive Anosov flows on 3-manifolds, to appear in C.B.M.S. lecture series.

[Fe1] S. Fenley, Asymptotic properties of depth one foliations in hyperbolic 3-manifolds, Jour. Diff. Geom. 36 (1992) 269-313. MR 93k:57030

[Fe2] S. Fenley, Quasi-isometric foliations, Topology 31 (1992) 667-676. MR 94a:57044

[Fe3] S. Fenley, Anosov flows in 3-manifolds, Ann. of Math. 139 (1994) 79-115. MR 94m:58162

[Fe4] S. Fenley, Quasigeodesic Anosov flows and homotopic properties of flow lines, Jour. Diff. Geom. 41 (1995) 479-514. MR 96f:58118

[Fe5] S. Fenley, Continuous extension of Anosov foliations in 3-manifolds with negatively curved fundamental group, to appear in Pacific J. Math.

[Fe6] S. Fenley, Homotopic indivisibility of closed orbits of Anosov flows in dimension 3, Math. Zeit. 225 (1997), 289-294. CMP 97:16

[Fe7] S. Fenley, One sided branching in Anosov foliations, Comm. Math. Helv. 70 (1995) 248-266. MR 96c:57052

[Fe8] S. Fenley, The structure of branching in Anosov flows of 3-manifolds, Comment. Math. Helv. (1998).

[Fe-Mo] S. Fenley and L. Mosher, Quasigeodesic flows in hyperbolic 3-manifolds, preprint.

[Gh] E. Ghys, Flots d'Anosov sur les 3-variétés fibrés en cercles, Ergod. Th. Dyn. Sys. 4 (1984) 67-80. MR 86b:58098

[Gh-Ha] E. Ghys and P. de la Harpe, eds., Sur les groupes hyperboliques d'aprés Mikhael Gromov, Progress in Math., 83 Birkháuser, 1991. MR 92f:53050

[Go] S. Goodman, Dehn surgery and Anosov flows, in Proceedings of the geometric dynamics conference, Lecture notes in mathematics 1007, Springer, 1983, 300-307. MR 84m:58003

[Gr] M. Gromov, Hyperbolic groups, in Essays on group theory, 75-263, Springer, 1987. MR 89e:20070

[Mor] J. Morgan, On Thurston's uniformization theorem for 3 dimensional manifolds, in The Smith conjecture, ed. by J. Morgan and H. Bass, Academic Press, 1984, pp. 37-125. MR 86i:57002

[Mo1] L. Mosher, Dynamical systems and the homology norm of a 3-manifold I. Efficient interesection of surfaces and flows, Duke Math. Jour. 65 449-500 (1992). MR 93g:57018a

[Mo2] L. Mosher, Dynamical systems and the homology norm of a 3-manifold II, Invent. Math. 107 243-281 (1992). MR 93g:57018b

[Mo3] L. Mosher, Examples of quasigeodesic flows on hyperbolic 3-manifolds, Topology '90 (Columbus, OH, 1990), Ohio State Univ. Math. Res. Inst. Publ., vol. 1, de Gruyter, Berlin, 1992, pp. 227-241. MR 93i:58120

[No] S. P. Novikov, Topology of foliations, Trans. Moscow Math. Soc. 14 (1963) 268-305. MR 34:824

[Pa] F. Palmeira, Open manifolds foliated by planes, Ann. of Math., 107 (1978) 109-131. MR 58:18490

[Pl1] J. Plante, Anosov flows, Amer. J. of Math., 94 (1972) 729-754. MR 51:14099

[Pl2] J. Plante, Anosov flows, transversely affine foliations and a conjecture of Verjovsky, J. London Math. Soc. (2) 23 (1981) 359-262. MR 82g:58069

[Pl3] J. Plante, Solvable groups acting on the line, Trans. A. M. S. 278 (1983) 401-414. MR 85b: 57048

[Sm] S. Smale, Differentiable dynamical systems, Bull. Amer. Math. Soc. 73 (1967) $747-817$. MR 37:3598

[Su] D. Sullivan, Cycles for the dynamical study of foliated manifolds and complex manifolds, Invent. Math. 36 (1976) 225-255. MR 55:6440

[Th1] W. Thurston, The geometry and topology of 3-manifolds, Princeton University Lecture Notes, 1982.

[Th2] W. Thurston, 3-dimensional manifolds, Kleinian groups and hyperbolic geometry, Bull. Amer. Math. Soc. (N.S.) 6 (1982) 357-381. MR 83h:57019

Department of Mathematics, Washington University, St. Louis, Missouri 63130

Department of Mathematics, Princeton University, Princeton, New Jersey 085441000

E-mail address: fenley@math.princeton.edu 